\title{
Enteric Opportunistic Infection and the Impact of Antiretroviral Therapy among HIV/AIDS Patients from Tehran, Iran
}

\author{
Hossein MASOUMI-ASL ${ }^{1}$, *Khadijeh KHANALIHA ${ }^{1}$, Farah BOKHARAEI-SALIM ${ }^{2}$, \\ Abdoulreza ESTEGHAMATI ${ }^{1}$, Saeed KALANTARI ${ }^{3}$, Maryam HOSSEINYRAD ${ }^{4}$
}

\section{Research Center of Pediatric Infectious Diseases, Institute of Immunology and Infectious Diseases, Iran University of Medical Sciences, Tebran, Iran \\ 2. Department of Virology, School of Medicine, Iran University of Medical Sciences, Tebran, Iran}

3. Department of Infectious Diseases, Rasoul-e-Akram Hospital, Iran University of Medical Sciences, Tehran, Iran

4. Consult Center of Behavior Diseases, West Health Center, Iran University of Medical Sciences, Tehran, Iran

*Corresponding Author: Email: khanaliha.kh@iums.ac.ir

(Received 10 Jan 2018; accepted 17 Apr 2018)

\begin{abstract}
Background: Opportunistic parasites have been identified as human pathogens, especially in immunodeficient patients. Microsporidian and coccidian infections cause chronic diarrhea as common clinical manifestation in HIV positive patients. In this study, the frequency of opportunistic infections, including microsporidian and coccidian infections, was evaluated in HIV/AIDS patients from Tehran and phylogenic analysis was performed for E. bieneusi isolates from these patients.

Methods: One hundred and two stool samples were collected from confirmed HIV/AIDS patients, referred to Consult Center of Behavior Diseases, West Health Center, Iran University of Medical Sciences in Tehran, Iran. The samples were transferred to Research Center of Pediatric Infectious Diseases, Institute of Immunology and Infectious Diseases, Iran University of Medical Sciences from Jan 2016 to Dec 2016. After conventional formalin-ether concentration, aniline blue staining method and acid-fast staining technique were used for detection of microsporidian spores and Cyclospora oocysts. DNA was extracted and nested PCR was performed. Results: Two $(1.96 \%)$ cases were found to be positive for intestinal microsporidia infection using aniline blue staining method and were confirmed as E. bieneusi by nested PCR. One patient was found with Cyclospora cayetanensis infection by acid-fast staining method and PCR. Giardia lamblia and Blastocystis hominis were detected as non-opportunistic parasites in 1/102 (0.98\%) and 2/102 (1.96\%) of the HIV positive patients, respectively. Conclusion: With respect to the use of antiretroviral therapy (ART) in HIV positive patients, we found a low frequency of infection.
\end{abstract}

Keywords: Opportunistic infection; HIV; Coccidian; Microsporidian

\section{Introduction}

Opportunistic parasites including microsporidian and coccidian infections have been identified as human pathogens, especially in immunodeficient individuals like organ transplant recipients (1), cancer patients (2), and HIV/AIDS patients (3). However, there are some reports of microsporidial infection in immunocompetent people also, especially those like travelers, chil- 
dren, and healthy human population from all over the world $(4,5)$.

The most common microsporidial infections are Enterocytozoon bieneusi and Encephalitozoon intestinalis in humans (6). E. bieneusi is a zoonotic pathogen that infects animals and causes infection in humans $(7,8)$. E. bieneusi infection causes chronic diarrhea and biliary illness and it is has been reported in individuals infected with HIV (4). Prevalence rate of E. bieneusi has been reported between 2.5\%-51\% for HIV-seropositive adult patients with diarrhea (9-11) and $4.6 \%$ for patients without diarrhea (12).

Diagnosis of microsporidia is based on the identification of spores by staining methods like chromotrope 2R, aniline blue and calcofluor white $(13,14)$, and various molecular methods (3, 12, 15).

Cyclospora cayetanensis infection has recently emerged as one of the opportunistic infections with worldwide distribution. A number of outbreaks have been reported in the United States and Canada (16). The oocyst of C. cayetanensis has been identified in the feces of immunocompetent people who travel to developing countries or consume contaminated fruits or salads, and in patients with AIDS. In Pune, India, Cyclospora was reported in $0.7 \%-3.3 \%$ of HIV positive individuals with diarrhea (17).

Oocysts of Cyclospora can be distinguished by direct examination of wet smear. They can also be observed by acid-fast staining method and diagnosis of oocysts sporulation after preservation in dichromate potassium $(2.5 \%)$ can be made (16, 18).

This study aimed to evaluate the frequency of opportunistic infections, including microsporidian and coccidian infections, among HIV/AIDS patients in Tehran.

\section{Methods}

In this cross-sectional study, 102 stool samples were collected from confirmed HIV/AIDS patients, with or without diarrhea, referred to Consult Center of Behavior Diseases, West Health
Center, Iran University of Medical Sciences, Tehran, Iran. The stool samples were transferred to Research Center of Pediatric Infectious Diseases, Institute of Immunology and Infectious Diseases, Iran University of Medical Sciences, from Jan 2016 to Dec 2016.

Wet mount smear was prepared from concentrated samples and all samples were observed under light microscope. Acid-fast staining was carried out for all samples and slides were observed and evaluated for detecting Cyclospora and another coccidian like cryptosporidium oocysts.

\section{Ethical issues}

This study was approved by the Ethics Committee of Iran University of Medical Sciences in accordance with the Helsinki Declaration and guidelines and all human participation has been obtained in accordance with informed consent.

\section{Cyclospora}

The stool was evaluated by direct examination of wet smear and conventional formalin-ether concentration technique was performed, smear was prepared and fixed with methanol. Next, the slide was stained with modified acid-fast staining method (18). The stool was washed in Phosphate-buffered saline (PBS) and preserved in dichromate potassium $(2.5 \%)$ for following up oocyst sporulation, and finally, autofluorescence of oocyst was observed with immunofluorescence microscope for further confirmation.

DNA extraction was performed by (Roche Diagnostics $\mathrm{GmbH}$, Mannheim, Germany) according to the manufacturer's instructions, with some modifications.

All primers used for $C$. cayetanensis were used from previously defined regions of the $18 \mathrm{~S}$ ribosomal RNA gene in C. cayetanensis. PCR was performed in a $25 \mu \mathrm{L}$ mixture containing the template $(3 \mu \mathrm{L}$ of DNA), $2.5 \mathrm{U}$ of Taq DNA polymerase, $2.5 \mu \mathrm{L}$ of $10 \mathrm{x}$ PCR buffer, 20 pmol of each primer, $100 \mu \mathrm{mol} \mathrm{dNTPs,} \mathrm{and} 0.15 \mathrm{mmol}$ $\mathrm{MgCl}_{2}$.

Nested PCR was performed by initial denaturation of 5 min at $95^{\circ} \mathrm{C}$, followed by a cycling program consisting of 35 cycles of denaturation at 94 
${ }^{\circ} \mathrm{C}$ for $30 \mathrm{sec}$, annealing at $53{ }^{\circ} \mathrm{C}$ for $30 \mathrm{sec}$, and extension at $72{ }^{\circ} \mathrm{C}$ for $90 \mathrm{sec}$. A final extension at $72{ }^{\circ} \mathrm{C}$ for $10 \mathrm{~min}$ was followed. A 636-bp product was expected after this round of PCR. The second round was similar to the first one, with the exception that the annealing temperature was $60{ }^{\circ} \mathrm{C}$. The inner primers were expected to produce a 294-bp product in case of the presence of C. cayetanensis DNA as the template.

Nested PCR for the detection of Cyclospora was performed using the outer primers F1E (5' -TACCCAATGAAAACAGTTT-3') and R2B (5'-CAGGAGAAGCCAAGGTAGG-3'). The inner primers used were F3E (5'CCTTCCGCGCTTCGCTGCGT-3') and R4B (5'-CGTCT'TCAAACCCCCTACTG-3') (15).

\section{E. bieneusi}

Formalin-ether concentration method was performed and after 5 min of methanol fixation, aniline blue staining was done for the detection of microsporidial spores (13).

DNA extraction was performed by (Roche Diagnostics $\mathrm{GmbH}$, Mannheim, Germany) according to the manufacturer's instructions and nested PCR was performed using E. bieneusi specific primers, designed based on the small subunit (SSU) of rRNA gene.

The nested PCR was done using a set of primers that were specific for E. bieneusi ITS region as well as a portion of the flanking large and small subunit ribosomal RNA genes (400 bp). The outer primers were EBITS3 (5'-GGTCATAGGGATGAAGAG-3') and EBITS4 (5'-T'TCGAGT'TCTTTCGCGCTC$\left.3{ }^{\prime}\right)$, and the inner primers were EBITS1 (5'GCTCTGAATATCTATGGCT-3') and EBITS2.4 (5'ATCGCCGACGGATCCAAGTG-3'). Finally, these reactions produced a fragment of $389 \mathrm{bp}$ (12).

The reaction mixture $(25 \mu \mathrm{L})$ contained $0.15 \mathrm{mM}$ $\mathrm{MgCl} 2,2.5 \mu \mathrm{L}$ of $10 \mathrm{x}$ PCR buffer, $100 \mu \mathrm{M}$ dNTPs, 20 pmol of each primer, and $2.5 \mathrm{U}$ of Taq DNA polymerase. PCR was performed as follows: denaturation at $94{ }^{\circ} \mathrm{C}$ for $3 \mathrm{~min}$, followed by 35 cycles of amplification (denaturation at $94{ }^{\circ} \mathrm{C}$ for $30 \mathrm{sec}$, annealing at $57^{\circ} \mathrm{C}$ for $30 \mathrm{sec}$, and elongation at $72{ }^{\circ} \mathrm{C}$ for $40 \mathrm{sec}$ ), and a final extension at $72{ }^{\circ} \mathrm{C}$ for $10 \mathrm{~min}$. Conditions for the secondary PCR were identical to that of the primary PCR, except that only 30 cycles were carried out at an annealing temperature of $55^{\circ} \mathrm{C}$. These reactions produced fragments of 435 and 389 bp, respectively (12).

Finally, PCR products were electrophoresed on $2 \%$ agarose gel. Negative and positive controls were included in all sets of PCRs.

\section{Sequencing}

The second round of PCR was performed with inner primers and PCR products were purified using the High Pure PCR Product Purification Kit (Roche Diagnostic, Mannheim, Germany) according to the manufacturer's instructions and were used for direct sequencing using the dye termination method and an ABI 3730xl sequencer (19).

\section{Results}

Out of $102 \mathrm{HIV}$ positive patients, 70 (68.6\%) were males and $32(31.4 \%)$ were females including 9 patients with CD4 T-cell count $<200$ cells / $\mu$ l, 17 patients with CD4 200-500 cells/ $\mu$ l, and 76 patients with CD $4>500$ cells $/ \mu$ l. The mean age of patients was $31 \mathrm{yr}$ (range of 19-48 yr). Among 102 stool samples, two $(1.96 \%)$ cases were found to be positive for intestinal microsporidia infection using aniline blue staining method and were confirmed as E. bieneusi by nested PCR. One patient $(0.98 \%)$ was found with $C$. cayetanensis infection by acid-fast staining method and PCR. G. lamblia and B. hominis were detected as non-opportunistic parasites in $1 / 102$ $(0.98 \%)$ and $2 / 102(1.96 \%)$ of the HIV positive patients, respectively (Table 1 ).

Microsporidian spores with aniline blue staining were oval and measured 1-1.5 $\mu \mathrm{m}$ in size (Fig. 1). The positive samples showed positive results for nested PCR for E. bieneusi also. A band of 389 bp corresponding to E. bieneusi was observed in positive cases (Fig. 2). These two positive E. bieneusi patients had chronic diarrhea as main clinical manifestation and showed a CD4 count of less than 200 cells / $\mu$ l. 
Table 1: Enteric opportunistic and non-opportunistic parasites among HIV/AIDS patients

\begin{tabular}{|c|c|c|c|c|}
\hline Parasites & $\begin{array}{c}C D 4>500 \\
\text { cells } / \mu 1 \\
N=76\end{array}$ & $\begin{array}{c}\text { CD4 200-500 } \\
\text { cells } / \mu 1 \\
N=17\end{array}$ & $\begin{array}{c}C D 4<200 \\
\text { cells } / \mu 1 \\
N=9\end{array}$ & $\begin{array}{c}\text { Total } \\
N(\%)\end{array}$ \\
\hline E. bieneusi & & & 2 & 2(1.96) \\
\hline Cyclospora cayetanensis & & & 1 & $1(0.98)$ \\
\hline Giardia lamblia & 1 & & & $1(0.98)$ \\
\hline Blastocystis hominis & 1 & 1 & & $2(1.96)$ \\
\hline Total & $2(1.96 \%)$ & $1(0.98 \%)$ & $3(2.9 \%)$ & $6 / 102(5.9)$ \\
\hline
\end{tabular}

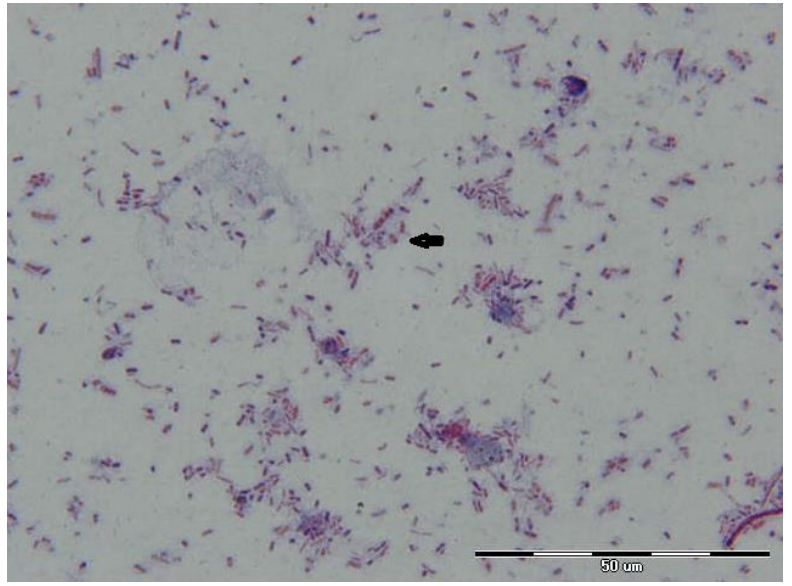

Fig. 1: Appearance of E. bieneusi spores in stool samples collected from HIV positive Patients by aniline blue staining method, 1000x magnification (origin picture)

The result of sequencing of two E. bieneusi positive patients in this study based on SSU rRNA gene ITS region demonstrated $99 \%$ identity with E. bieneusi isolates HNSC9, 178, HNZM19 and Chan L46, and 98\% identity with isolates HNZM54, 356, and 601.

The sequences were deposited in GenBank with accession numbers MF163429 and MF163430 (Fig. 3).

C. cayetanensis oocysts were found in a HIV positive patient with watery diarrhea and CD4 count less than 200 cells/ $\mu$ l. The oocysts were almost 8-10 $\mu \mathrm{m}$ in diameter and partial acid-fast positive with wrinkled edge in some oocyst cases (Fig. 4). The result of preservation in dichromate potassium $(2.5 \%)$ and oocysts sporulation showed that oocysts had two sporocysts and each sporocyst contained two sporozoites.

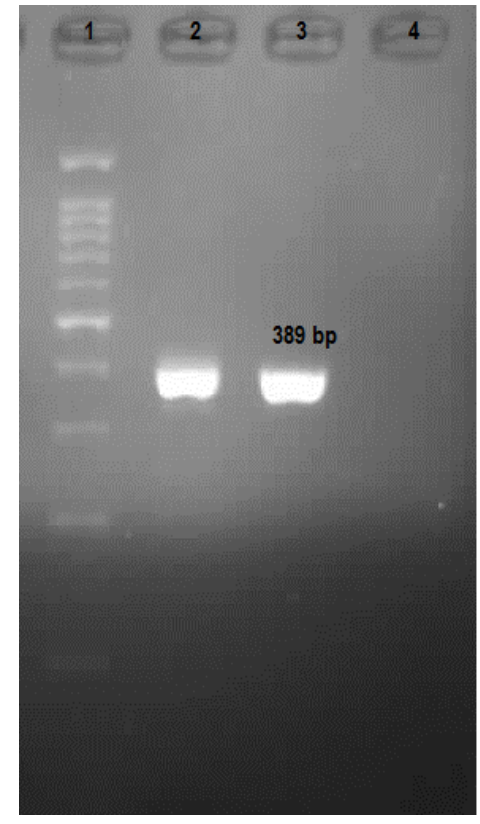

Fig. 2: Agarose gel Electrophoresis of PCR products of E. bieneusi in Iranian HIV positive patients.1:100 bp DNA ladder marker; 2-3 Positive E. bieneusi patients (389 bp); 4: Negative Samples

For further confirmation, autofluorescence of oocyst was observed under immunofluorescence microscope.

C. cayetanensis was also confirmed by nested PCR. A band of 294 bp corresponding to Cyclospora was observed in positive cases (Fig. 5). The result of sequencing and blast showed $99 \%$ identity with C. cayetanensis isolates H6 (1-4), PC1, and HMCCPR2. The result of $18 \mathrm{~S}$ ribosomal rRNA sequences of $C$. cayetanensis described in this paper was deposited in GenBank with the accession number KY769936. 


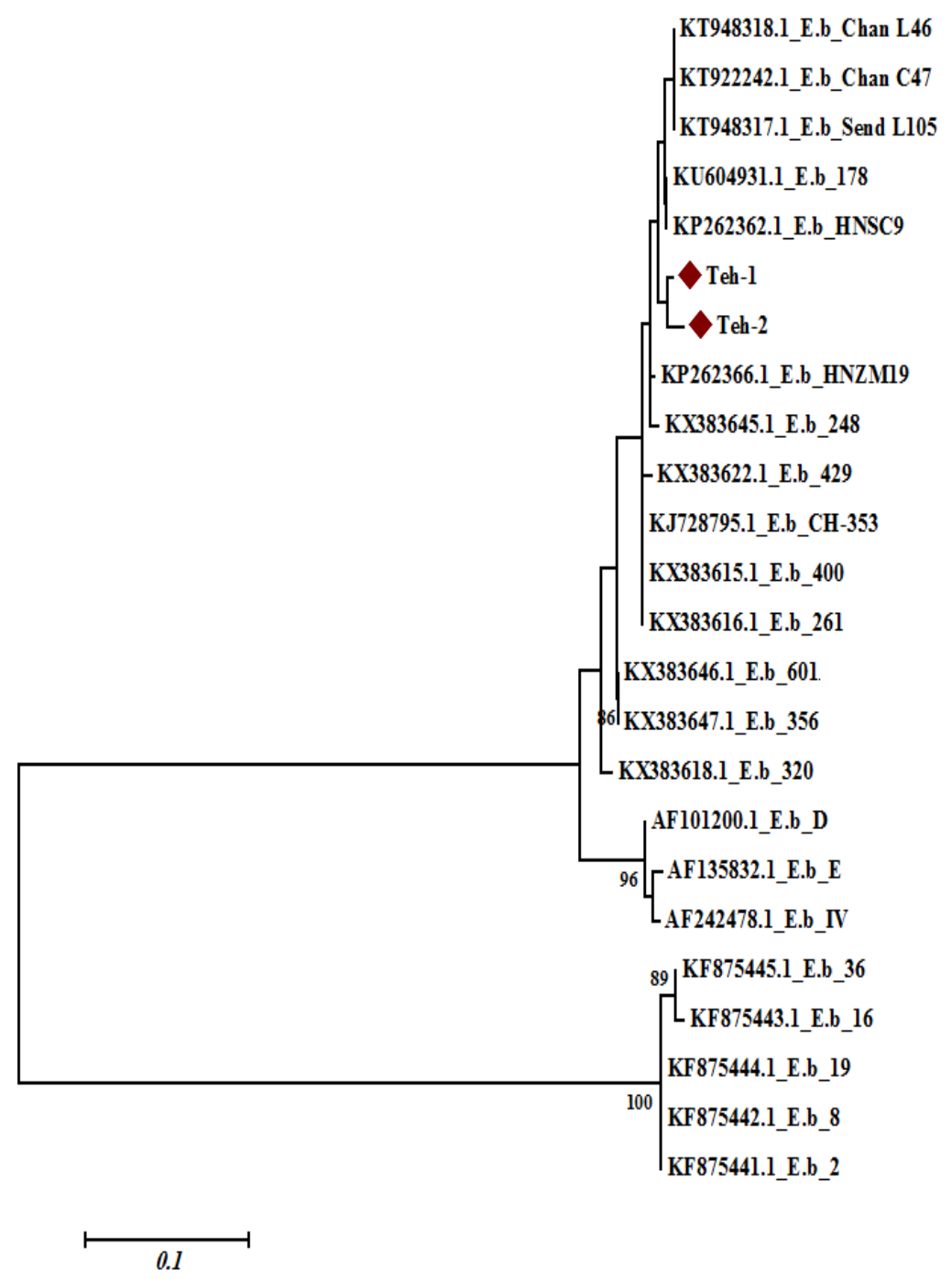

Fig. 3: Phylogenetic tree based on E. bieneusi nucleotide sequences from two individuals with E. bieneusi infection and those corresponding to different E. bieneusi genotypes taken from the GenBank database. Bootstrap values $\geq 70$ achieved after 1000 replicates are shown at the nodes

G. lamblia and B. hominis were detected as nonopportunistic parasites in $1 / 102(0.98 \%)$ and 2/102 (1.96\%) HIV positive patients with CD4 cell count more than 200 cells / $\mu$ lin this study.

\section{Discussion}

Opportunistic infections are found frequently in HIV positive patients, especially when the CD4 T-cell count is less than 200 cells / $\mu \mathrm{l}(3)$.

Microsporidiosis in HIV positive patients have been reported from Southeast Asia (India, Thai- land), the Middle-East (Turkey), Europe, Africa (Tunisia, Mali, Uganda, Senegal, Zimbabwe), and Latin America (Brazil, Peru) (20).

Diagnosis of E. bieneusi infection is based on staining methods and visualization by light microscopy and electron microscopy, and PCR methods. The comparison of results obtained is difficult because of the differences between these methods (21). Prevalence of E. bieneusi in HIV positive patients with diarrhea in developed countries like Europe, Australia, and North America has been reported to be between $2 \%$ - 
$78 \%$ (21-23), however, this rate was reported from $1.4 \%-4.3 \%$ in HIV positive patients without diarrhea (24).

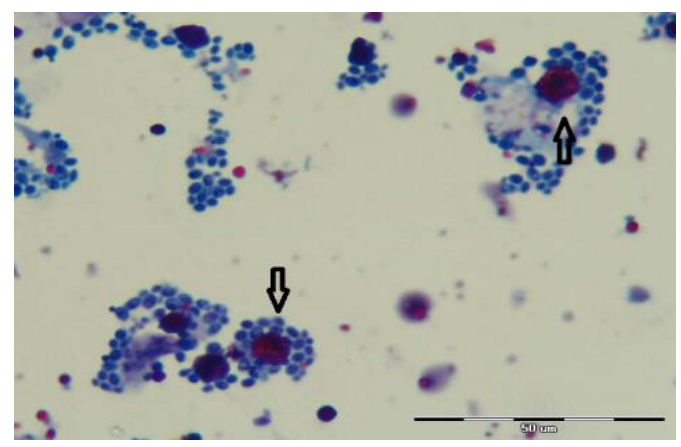

Fig. 4: Appearance of C. cayetanensis oocysts in Stool samples collected from HIV positive patients by modified acid fast staining method, 1000x magnification (origin picture)

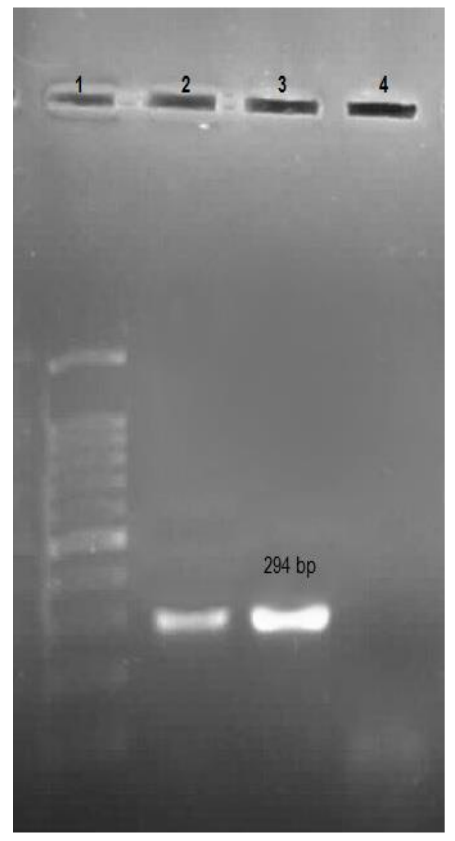

Fig. 5: Agarose Gel Electrophoresis of PCR products of C. cayetanensis in Iranian HIV positive patient .1:100 bp DNA ladder marker; 2: Positive $C$. cayetanensis $\mathrm{Pa}-$ tient; 3: Positive control (294 bp). 4: Negative sample

In this study, $102 \mathrm{HIV}$ positive patients were evaluated to assess the occurrence of protozoa opportunistic infection and two cases were shown to be E. bieneusi positive by staining and nested PCR. Both of these patients had chronic diarrhea. Sequencing confirmed the isolates as $E$. bieneusi, with $99 \%$ identity to E. bieneusi isolates HNSC9, 178, HNZM19, and Chan L46; and 98\% identity with isolates HNZM54, 356, and 601.

Prevalence of enteric E. bieneusi in HIV positive patients has been reported in various countries, like Russia (1.2\%) (25), Thailand (5.6\%) (26), Vietnam $(7.1 \%)(27)$, Nigeria $(2.6 \%)(28)$, Switzerland $(12.7 \%)$ in patients with diarrhea and $0.4 \%$ in patients without diarrhea (29), Holland $(7.7 \%)$ (30), UK (8.3\%) (31), Peru (3.9\%) (32) using fecal samples by PCR method.

In a study, from $356 \mathrm{HIV}$ positive patients, eight $(2.2 \%)$ cases were identified as E. bieneusi by staining method and PCR in Shiraz (3).

In a study conducted in North India, E. bieneusi was reported in $2.5 \%$ of the HIV-infected individuals. Furthermore, both $C$. cayetanensis and $B$. hominis were detected in 3.3\% of the patients (9).

A systematic review and meta-analysis had reported the overall prevalence of microsporidia infection in immunocompromised patients to be $8.18 \%$ in Iran. In addition, prevalence of microsporidia infection was $15.4 \%$ in immunocompromised patients with chronic diarrhea, $4.1 \%$ in patients without diarrhea, and $12.9 \%$ in patients with CD4 less than 200 cells/ $\mu$ (33).

Some studies carried out in developed countries demonstrated that the prevalence of E. bieneusi in HIV positive patients is decreasing with the use of highly active antiretroviral therapy (HAART) $(29,34)$.

Prevalence of intestinal parasites in pre-ART and patients with ART treatment was 39\% and $17.6 \%$, respectively. All positive cases related to Cryptosporidium spp were found in the pre-ART group and significantly related to $\mathrm{CD} 4$ $<200$ cells/ $\mu \mathrm{L}$ (35).

Prevalence of intestinal parasites in HIV/AIDS positive patients was compared between preHAART and HAART groups in Brazil. Frequency of Isospora belli, Cryptosporidium sp. and G. lamblia were (4.8\% and 1\%), (8.1\% and 0$)$ and $(7.9 \%$ and $1 \%)$ in pre-HAART and HAART treatment respectively that may due to effect of HAART treatment on intestinal parasites (36). Effect of antiretroviral therapy on opportunistic 
infection like cryptosporidiosis and microsporidiosis in HIV positive patients were evaluated. The result confirmed combination antiretroviral therapy is able to improve the course of cryptosporidiosis and microsporidiosis in HIV-1 positive patients (37). HAART treatment in HIV positive patients in Australia has led to a decrease in microsporidial infections from $11 \%$ in 1995 to $0 \%$ in 2004 (38).

Contrary to this, prevalence of intestinal parasites remained high in pre- ART in comparision with on-ART pateints $84.6 \%$ and $82.3 \%$ respectively. Opportunistic infection like C. parvum, Microsporidium spp. and $I$. belli were associated with CD4 <200cells/ $\mu \mathrm{L}$ in on-ART (39).

HIV/AIDS patients referred to Consult Center of Behavior Diseases, West Health Center, Iran University of Medical Sciences in Tehran, were used for the analysis. Two cases of E. bieneusi among 102 HIV positive patients were found with chronic diarrhea as main clinical manifestation. Furthermore, low prevalence of E. bieneusi in this study was interpreted by administration of ART treatment in all patients and use sulfamethoxazole-trimethoprim treatment during the course of AIDS for patients with CD4 T-cell count less than 200 cells/ $\mu$ l.

C. cayetanensis is a coccidian protozoan observed in many different countries. There are some reports about presence of $C$. cayetanensis in HIV positive patients from Argentina (40), Turkey (41), and Iran (18). Prevalence of C. cayetanensis has been reported to be 3\% in Cuba (42), 11\% in Haiti (43), between $0.7 \%-3.3 \%$ in India $(9,17)$, $2.2 \%$ in Thailand (44), and $1 \%$ in Tanzania (45).

In this study, one $C$. cayetanensis positive sample was detected in 102 stool samples collected from HIV positive patients. The sequencing of $C$. cayetanensis and blast alignment showed $99 \%$ identity with C. cayetanensis isolates H6 (1-4), PC1, and HMCCPR2.

No Cryptosporidium spp. was found from 102 stool samples collected from HIV positive patients by modified acid-fast staining method and G. lamblia and $B$. hominis as non-opportunistic parasites were detected in $1 / 102(0.98 \%)$, and $2 / 102(1.96 \%)$
HIV positive patients with CD4 T-cell count more than 200 cells/ $\mu$ l.

Some studies have reported a prevalence range from $(1.5 \%-9.4 \%)$ of enteric coccidian, G. lamblia with prevalence range $(3.1 \%-7.3 \%)$ and B. hominis $(4.4 \%)$ as most common non-opportunistic parasites in HIV/AIDS patients in Iran $(46,47)$.

In this study, E. bieneusi and C. cayetanensis cases were detected from HIV positive patients as opportunistic infection. Phylogenetic mapping indicated that these Iranian E. bieneusi isolates were most closely related to E. bieneusi isolates HNSC9, 178, HNZM19, and Chan L46; and C. cayetanensis isolates showed identity with $C$. cayetanensis isolates H6 (1-4), PC1, and HMCCPR2.

\section{Conclusion}

In this study, with the use of ART treatment in HIV positive patients and administration of sulfamethoxazole-trimethoprim treatment during the course of AIDS for patients with CD4 T-cell counts less than 200 cells/ $\mu$ l, frequency of microsporidian and coccidian infection was low.

\section{Ethical considerations}

This study was approved by the Ethics Committee of Iran University of Medical Sciences code number (IR.IUMS.REC1394-01-131-25831). Ethical issues (Including plagiarism, informed consent, misconduct, data fabrication and/or falsification, double publication and/or submission, redundancy, etc.) have been completely observed by the authors.

\section{Acknowledgements}

This study was funded by Research Center of Pediatric Infectious Diseases, Iran University of Medical Sciences in Tehran, Iran with Grant number (94-01-131-25831).

\section{Conflict of interest}

The authors declare that there is no conflict of interests. 


\section{References}

1. Agholi M, Hatam GR, Motazedian MH (2013). HIV/AIDS-associated opportunistic protozoal diarrhea. AIDS Res Hum Retroviruses, 29:35-41.

2. Agholi M, Hatam GR, Motazedian MH (2013). Microsporidia and coccidia as causes of persistence diarrhea among liver transplant children: incidence rate and species/genotypes. Pediatr Infect Dis J, 32(2):185-187.

3. Anane S, Attouchi H (2010). Microsporidiosis: epidemiology, clinical data and therapy. Gastroenterol Clin Biol, 34(8-9):450-464.

4. Bachur TPR, Vale JM, Coêlho ICB et al (2008). Enteric parasitic infections in HIV/AIDS patients before and after the highly active antiretroviral therapy. Braz J Infect Dis, 12(2):115-122.

5. Cegielski JP, Ortega YR, McKee S, Madden JF et al (1999). Cryptosporidium, Enterocytozoon, and Cyclospora infections in pediatric and adult patients with diarrhea in Tanzania. Clin Infect Dis, 28(2):314-321.

6. Chawla R, Ichhpujani R (2011). Enteric sporeforming opportunistic parasites in HIV/AIDS. Trop Parasitol, 1(1):15-19.

7. Conteas $\mathrm{CN}$, Berlin $\mathrm{O}$, LaRiviere $\mathrm{MJ}$ et al (1998). Examination of the prevalence and seasonal variation of intestinal microsporidiosis in the stools of persons with chronic diarrhea and human immunodeficiency virus infection. $A m J$ Trop Med Hyg, 58:559-561.

8. Coyle CM, Wittner M, Kotler DP et al (1996). Prevalence of microsporidiosis due to Enterocytozoon bieneusi and Encephalitozoon (Septata) intestinalis among patients with AIDS-related diarrhea: determination by polymerase chain reaction to the microsporidian small-subunit rRNA gene. Clin Infect Dis, 23:1002-1006.

9. Daryani A, Sharif M, Meigouni M et al (2009). Prevalence of intestinal parasites and profile of CD4+ counts in HIV+/AIDS people in north of Iran, 2007-2008. Pak J Biol Sci, 12(18):1277-1281.

10. Dengjel B, Zahler M, Hermanns W et al (2001). Zoonotic potential of Enterocytozoon bieneusi. J Clin Microbiol, 39(12):4495-4499.
11. Escobedo AA, Núñez FA (1999). Prevalence of intestinal parasites in Cuban acquired immunodeficiency syndrome (AIDS) patients. Acta Trop, 72(1):125-130.

12. Espern A, Morio F, Miegeville M et al (2007). Molecular study of microsporidiosis due to Enterocytozoon bieneusi and Encephalitozoon intestinalis among human immunodeficiency virus-infected patients from two geographical areas: Niamey, Niger, and Hanoi, Vietnam. J Clin Microbiol, 45(9):2999-3002.

13. Field A, Hing M, Milliken S, Marriott D (1993). Microsporidia in the small intestine of HIVinfected patients. A new diagnostic technique and a new species. Med J Aust, 158(6):390394.

14. Garcia LS, Bruckner DA (1993). Diagnostic medical parasitology. Washington, DC:131-135.

15. Ghaderipour M, Khanaliha K, Mohebali M et al (2017). Emerging Intestinal Microsporidia Infection in General Population in Jiroft District, Southeastern Iran: A Cross-sectional Study in 2013-2014. Iran J Public Health, 46(12):1697-1703.

16. Ghoyounchi R, Ahmadpour E, Spotin A et al (2017). Microsporidiosis in Iran: A systematic review and meta-analysis. Asian Pac J Trop Med, 10(4):341-350.

17. Khanaliha K, Masoumi-Asl H, Bokharaei-Salim F et al (2017). Double-stranded RNA viral infection of Trichomonas vaginalis (TVV1) in Iranian isolates. Microb Pathog, 109:56-60.

18. Khanaliha K, Mirjalali H, Mohebali M, Tarighi F, Rezaeian M (2014). Comparison of three staining methods for the detection of intestinal Microspora spp. Iran J Parasitol, 9(4): $445-451$.

19. Khanaliha K, Mohebali M, Davoudi S et al (2015). Detection of emergence Cyclospora cayetanensis in A HIV+/AIDS patient with diarrhea from Tehran: a case report. Iran J Public Health, 44(6): 865-868.

20. Kulkarni SV, Kairon R, Sane SS et al (2009). Opportunistic parasitic infections in HIV/AIDS patients presenting with diarrhoea by the level of immunesuppression. Indian J Med Res, 130(1):63-6.

21. Lono AR, Kumar S, Chye TT (2008). Incidence of microsporidia in cancer patients. $J$ Gastrointest Cancer, 39(1-4):124-129. 
22. Maggi P, Larocca AM, Quarto M et al (2000). Effect of antiretroviral therapy on cryptosporidiosis and microsporidiosis in patients infected with human immunodeficiency virus type 1. Eur J Clin Microbiol Infect Dis, 19(3):213-217.

23. Manatsathit S, Tansupasawasdikul S, Wanachiwanawin D et al (1996). Causes of chronic diarrhea in patients with AIDS in Thailand: a prospective clinical and microbiological study. J Gastroenterol, 31(4):533-537.

24. Matos O, Lobo ML, Xiao L (2012). Epidemiology of Enterocytozoon bieneusi infection in humans. $J$ Parasitol Res, 2012:981424.

25. Missaye A, Dagnew M, Alemu A, Alemu A (2013). Prevalence of intestinal parasites and associated risk factors among HIV/AIDS patients with pre-ART and on-ART attending dessie hospital ART clinic, Northeast Ethiopia. AIDS Res Ther, 10(1):7.

26. Mohandas K, Sehgal R, Sud A, Malla N (2002). Prevalence of intestinal parasitic pathogens in HIV-seropositive individuals in Northern India. Jpn J Infect Dis, 55(3):83-84.

27. Nsagha DS, Njunda AL, Assob NJC et al (2016). Intestinal parasitic infections in relation to CD4+ $\mathrm{T}$ cell counts and diarrhea in HIV/AIDS patients with or without antiretroviral therapy in Cameroon. BMC Infect Dis, 11;16:9.

28. Ojuromi OT, Izquierdo F, Fenoy S et al (2012). Identification and characterization of microsporidia from fecal samples of HIVpositive patients from Lagos, Nigeria. PLoS One, 7(4):e35239.

29. Orlandi PA, Lampel KA (2000). Extraction-free, filter-based template preparation for rapid and sensitive PCR detection of pathogenic parasitic protozoa. J Clin Microbiol, 38(6):22712277.

30. Pape JW, Verdier R-I, Boncy M et al (1994). Cyclospora infection in adults infected with HIV: clinical manifestations, treatment, and prophylaxis. Ann Intern Med, 121(9):654-657.

31. Polley SD, Boadi S, Watson J, Curry A, Chiodini PL (2011). Detection and species identification of microsporidial infections using SYBR Green real-time PCR. J Med Microbiol, 60(Pt 4):459-466.
32. Rinder H, Thomschke A, Dengjel B et al (2000). Close genotypic relationship between Enterocytozoon bieneusi from humans and pigs and first detection in cattle. J Parasitol, 86(1):185-188.

33. Ryan NJ, Sutherland G, Coughlan K et al B (1993). A new trichrome-blue stain for detection of microsporidial species in urine, stool, and nasopharyngeal specimens. J Clin Microbiol, 31(12):3264-3269.

34. Sak B, Brady D, Pelikánová M et al (2011). Unapparent microsporidial infection among immunocompetent humans in the Czech Republic. J Clin Microbiol, 49(3):1064-1070.

35. Saksirisampant W, Prownebon J, Saksirisampant $P$ et al S (2009). Intestinal parasitic infections: prevalences in HIV/AIDS patients in a Thai AIDS-care centre. Ann Trop Med Parasitol, 103(7):573-581.

36. Samie A, Obi C, Tzipori S, Weiss L, Guerrant R (2007). Microsporidiosis in South Africa: PCR detection in stool samples of HIV-positive and HIV-negative individuals and school children in Vhembe district, Limpopo Province. Trans $\mathrm{R}$ Soc Trop Med Hyg, 101(6):547-554.

37. Santín M, Trout JM, Vecino JAC, Dubey J, Fayer R (2006). Cryptosporidium, Giardia and Enterocytozoon bieneusi in cats from Bogota (Colombia) and genotyping of isolates. Vet Parasitol, 141(3-4):334-339.

38. Sarfati C, Bourgeois A, Menotti J et al (2006). Prevalence of intestinal parasites including microsporidia in human immunodeficiency virus-infected adults in Cameroon: a crosssectional study. Am J Trop Med Hyg, 74(1):162164.

39. Sobottka I, Schwartz DA, Schottelius J et al (1998). Prevalence and clinical significance of intestinal microsporidiosis in human immunodeficiency virus-infected patients with and without diarrhea in Germany: a prospective coprodiagnostic study. Clin Infect Dis, 26(2):475-480.

40. Sokolova OI, Demyanov AV, Bowers LC et al (2011). Emerging microsporidian infections in Russian HIV-infected patients. J Clin Microbiol, 49:2102-2108.

41. Sulaiman Im, Bern C, Gilman R et al (2003). A Molecular Biologic Study of Enterocytozoon 
bieneusi in HIV-Infected Patients in Lima, Peru. J Eukaryot Microbiol, 50:591-596.

42. Van Gool T, Snijders F, Reiss P et al (1993). Diagnosis of intestinal and disseminated microsporidial infections in patients with HIV by a new rapid fluorescence technique. J Clin Pathol, 46(8):694-699.

43. Van Hal S, Muthiah K, Matthews G et al (2007). Declining incidence of intestinal microsporidiosis and reduction in AIDSrelated mortality following introduction of HAART in Sydney, Australia. Trans R Soc Trop Med Hyg, 101(11):1096-100.

44. Velasquez J, Carnevale S, Cabrera M et al (2004). [Cyclospora cayetanensis in patients with
AIDS and chronic diarrhea]. Acta Gastroenterol Latinoam, 34(3):133-137.

45. Weber R, Ledergerber B, Zbinden R et al (1999). Enteric infections and diarrhea in human immunodeficiency virus-infected persons: prospective community-based cohort study. Arch Intern Med, 159(13):1473-1480.

46. Yazar S, Yalcln S, Sahin Í (2004). Human cyclosporiosis in Turkey. World J Gastroenterol, 10:1844.

47. Zali MR, Mehr AJ, Rezaian M et al (2004). Prevalence of intestinal parasitic pathogens among HIV-positive individuals in Iran. Jpn J Infect Dis, 57(6):268-70. 